\title{
Impulse Force Balance for Ultrashort Duration Hypersonic Test Facilities
}

\author{
P. Singh, ${ }^{1}$ V. Menezes, ${ }^{1}$ K. J. Irimpan, ${ }^{1}$ and H. Hosseini ${ }^{2}$ \\ ${ }^{1}$ Department of Aerospace Engineering, Indian Institute of Technology Bombay, Powai, Mumbai 400-076, India \\ ${ }^{2}$ Institute of Pulsed Power Science and Graduate School of Science and Technology, Kumamoto University, \\ Kumamoto 860-8555, Japan \\ Correspondence should be addressed to H. Hosseini; hosseini@kumamoto-u.ac.jp
}

Received 15 September 2014; Accepted 30 April 2015

Academic Editor: Anindya Ghoshal

Copyright (C) 2015 P. Singh et al. This is an open access article distributed under the Creative Commons Attribution License, which permits unrestricted use, distribution, and reproduction in any medium, provided the original work is properly cited.

\begin{abstract}
This paper presents the measurement of side force, pitching, and yawing moments on a model, using an accelerometer force balance, in a short duration hypersonic shock tunnel. The test model is a blunt-nosed, flapped delta wing, mounted on a support sting through a force balance. The flexible rubber bushes constituting the balance allow the model to float freely on the sting during the test. The accelerometers were located in the model to record accelerations in the directions of interest. The model was tested in shock tunnel at Mach 8 at different angles of incidence with the freestream. Dynamic calibration of the test assembly was carried out for the acquisition of impulse response functions for the above components of force and moments, using an impulse hammer. The convolution technique was applied to derive the impulse response functions. The accelerometer outputs from the model in the hypersonic freestream were processed using the respective impulse response functions to derive the unknown aerodynamic force and moments. The newly adopted convolution technique has been found very effective for data reduction from accelerometer force balances developed for shock tunnel applications.
\end{abstract}

\section{Introduction}

Impulse mode of operation is common in most of the groundbased hypersonic test facilities as the requirement on energy and thermal survival would exceed the system capabilities otherwise. Impulse mode of operation poses challenges of quick-response and time-oriented uncertainties during the measurements. Hence, the short duration measurement methodologies differ from their blow-down counterparts with respect to response-time, frequency, model-mounts, and data acquisition $[1,2]$. The hypersonic cruise vehicles have slender shapes equipped with control surfaces, which give rise to a high lift-to-drag ratio and good maneuverability that are essential for an efficient mission. Knowledge of forces and moments on hypersonic vehicles is critical in the decisions pertinent to the structure, stability, propulsive unit, and trajectory of the vehicle. The force balances are the most useful devices $[3,4]$ in such measurement tasks as these balances yield an integrated output corresponding to forces and moments in the desired directions. The aerodynamic forces and moments on a test model in a hypersonic shock tunnel can also be measured by surface pressure measurement [5] and visual [6] techniques, but the output of the force balances is free of the concerns of geometrical complexity, spatial resolution, and time-dependency. In this backdrop, we developed an accelerometer-based force balance to measure forces and moments on hypersonic lifting models in shock tunnels. The balance with the model was calibrated for its impulse response function for a range of known input. The acquired impulse response function of the system was then used to derive the forces and moments under unknown conditions, such as in a hypersonic freestream in the shock tunnel.

This paper presents the measurement of side force, pitching, and yawing moments on a blunt, flapped delta wing in a hypersonic freestream of Mach 8 , in a shock tunnel of approximately $1.5 \mathrm{~ms}$ test duration. The measurements were carried out at different angles of attack of the model ranging from 0 to 15 degrees, at intervals of 5 degrees. The objective of the work was to experimentally derive the side force, pitching, and yawing moments on the model 


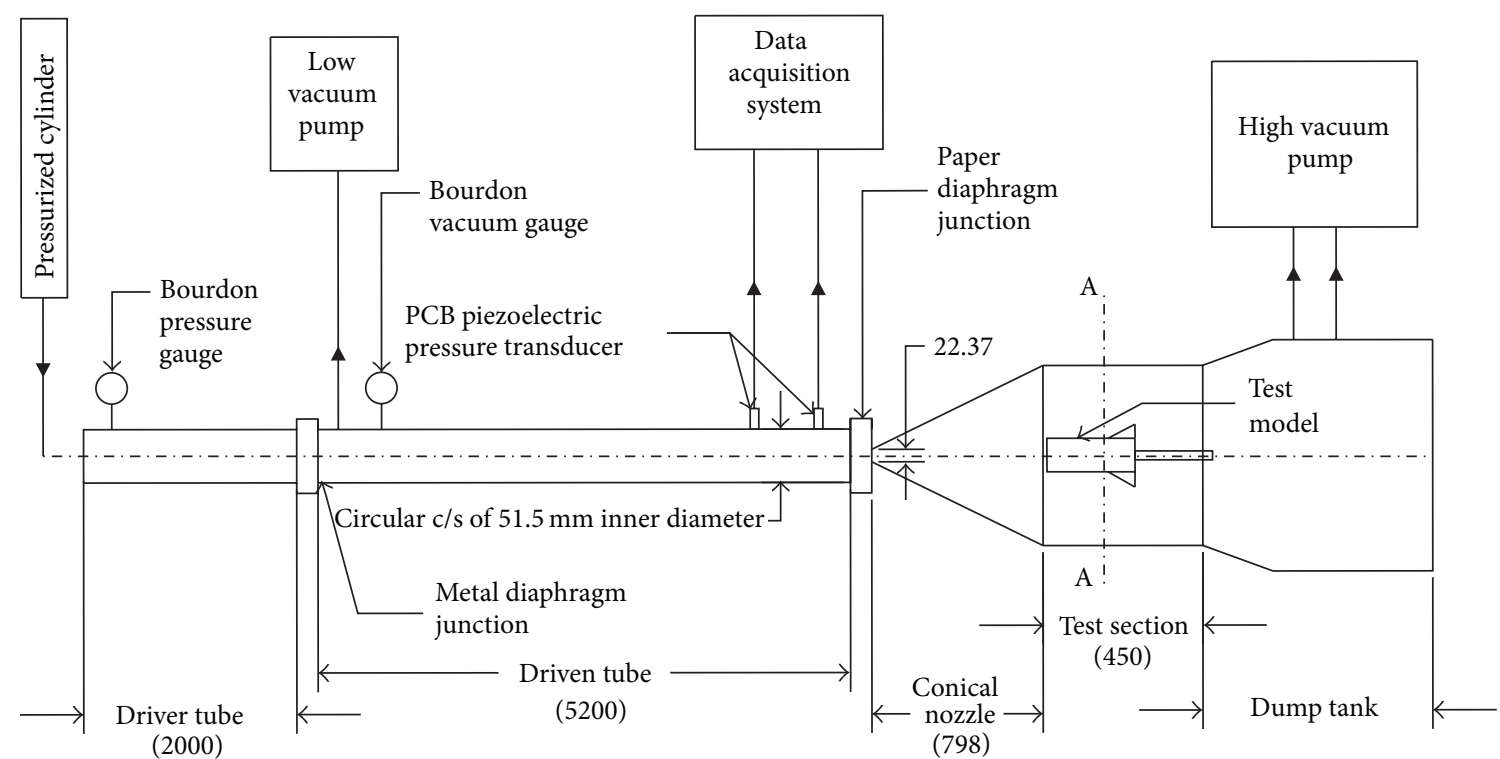

Section A-A: square c/s with $320 \mathrm{~mm}$ side

Dimensions ( $\mathrm{mm})$

FIGURE 1: Schematic of the hypersonic shock tunnel, IITB-ST.

TABLE 1: Freestream conditions for the tests in the shock tunnel.

\begin{tabular}{lccccr}
\hline Test gas & $P_{\infty}(\mathrm{Pa})$ & $T_{\infty}(\mathrm{K})$ & $M_{\infty}$ & Total Enthalpy $(\mathrm{MJ} / \mathrm{kg})$ & Reynolds no. $(/ \mathrm{m})$ \\
\hline Air & $50.6 \pm 3.74 \%$ & $55.4 \pm 3.74 \%$ & $8 \pm 2.5 \%$ & $0.768 \pm 3.74 \%$ & $1.04 \times 10^{6} \pm 3.63 \%$ \\
\hline
\end{tabular}

using an impulse response function of the system, where the construction of the model assembly, sensor uncertainties, restraint of the test model, and ultrashort duration effects do not influence the results. The results obtained by this method are believed to be more accurate in comparison with other data reduction methodologies [7].

\section{Experimental Methods}

2.1. Hypersonic Shock Tunnel. The experiments were performed in a hypersonic shock tunnel at IIT Bombay (IITBST) [8], which is presented schematically in Figure 1. The IITB-ST has a $50 \mathrm{~mm}$ diameter shock tube that is operated in a reflected-shock mode. The operation of the tube is initiated by the rupture of a thin aluminum diaphragm, which launches an incident shock wave into the test gas in the tube. The shock wave propagates through the test gas twice during the operation, once on incidence and the next on reflection from the tube end. The shock propagations compress and heat the test gas to high levels, before its expansion to a hypersonic freestream of Mach 8 through a converging-diverging nozzle of $22 \mathrm{~mm}$ and $300 \mathrm{~mm}$, inlet and exit diameters. The freestream conditions for the present set of experiments in the tunnel are presented in Table 1. Figure 2 presents the pressure traces at the inlet and exit of the converging-diverging nozzle of the tunnel during one of the experiments. The steady flow duration and the available

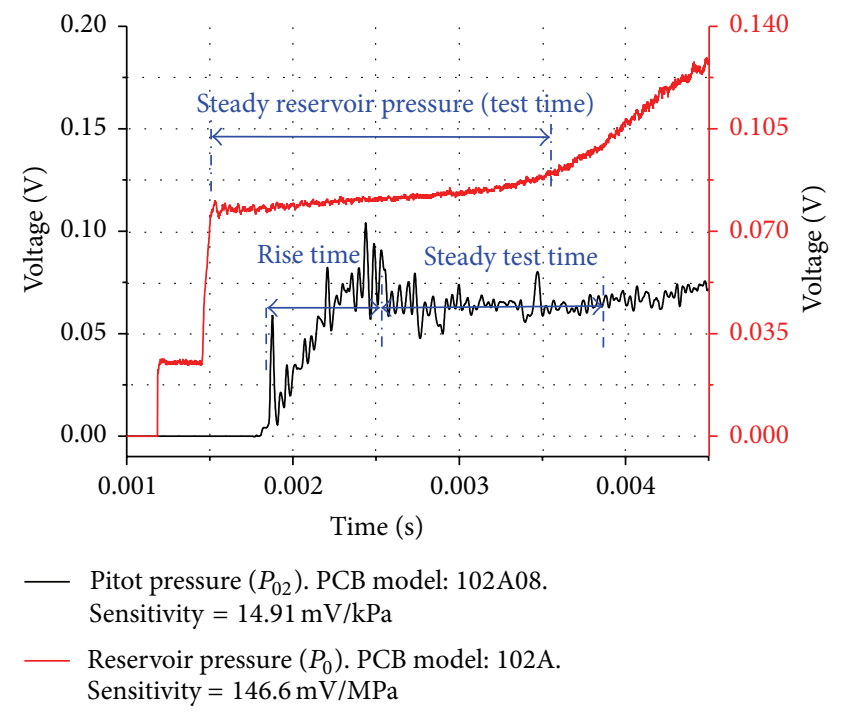

FIGURE 2: Representative pressure traces of the reservoir $\left(P_{0}\right.$, inlet of nozzle) and Pitot tube $\left(P_{02}\right.$, exit of nozzle) in the shock tunnel.

test time in the tunnel test section are indicated on the pressure signals. The pressure signals were acquired using the piezoelectric pressure transducers of PCB Piezotronics (USA). The model numbers and sensitivities of the pressure transducers are indicated in the figure. 


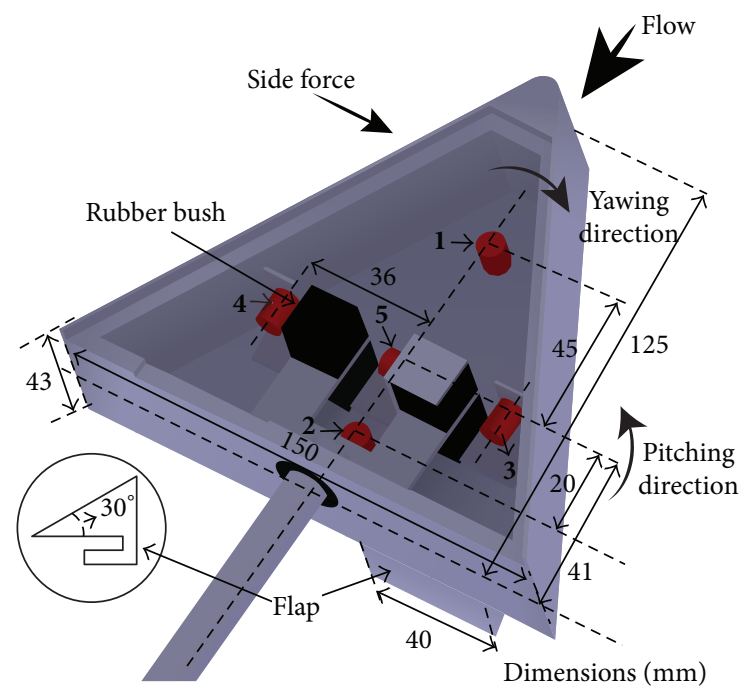

FIgURE 3: Schematic of the test model. Labels 1-5 indicate accelerometers. Accelerometer model (all) 352C67 of PCB Piezotronics (USA). Sensitivity $\approx 100 \mathrm{mV} / \mathrm{g}$.

2.2. Test Model and Force Balance. The test model is a bluntnosed delta wing with rectangular flaps deployed on its trailing edge. The model is a lifting body, which generated all the aerodynamic forces and moments when arranged at an angle of incidence to the freestream. The model was made of aluminum and weighed about $0.52 \mathrm{~kg}$, including the sensors and their mounts. The model had its Center of Gravity (CG) located at $41 \mathrm{~mm}$ (measured along the longitudinal axis) from the base. The location of CG was determined experimentally by balancing the model horizontally on a pointed $L$ shaped lever. The measurement of the first three components, namely, drag, lift, and rolling moment on the model, was reported in [9]. The present phase of research focused on the measurement of side force, pitching, and yawing moments. The schematic of the test model is presented in Figure 3. The orientation of the accelerometers in the assembly is indicated in the schematic. The model-balance-assembly (the system) was suspended on four rubber bushes, which allowed the system to float freely during the operations so that the accelerations corresponding to different maneuvers could be sensed by the accelerometers. The bush material was flexible nitrile foam (NBR) rubber with a density of $85 \mathrm{~kg} / \mathrm{m}^{3}$. The bushes were cubic with a dimension of $12 \mathrm{~mm}$ and had a stiffness of $3,367 \mathrm{~N} / \mathrm{m}$ in compression, $10,753 \mathrm{~N} / \mathrm{m}$ in tension, and $2,632 \mathrm{~N} / \mathrm{m}$ in shear. The bushes were chosen such that they deformed under the applied aerodynamic loads in the tunnel, making the model unrestrained during the tests so that the accelerometers could pick up the model accelerations with minimal ripples/vibrations. There had to be a tradeoff between the bush stiffness and the model unrestraint. Five uniaxial accelerometers were used in the system to capture five accelerations of the model due to pitching, yawing, and side force during the aerodynamic tests. The accelerometers were sensitive only along their longitudinal axes and were insensitive to accelerations in other directions. The accelerometers were piezoelectric and were from $\mathrm{PCB}$

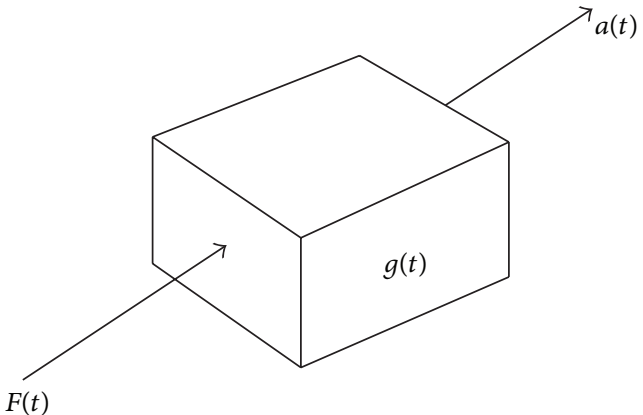

FIGURE 4: Schematic representing a linear, time-invariant system.

Piezotronics (USA) (model 352C67), with a thread-mounting on the base. The entire test assembly/system was light and rigid and had a high natural frequency higher than the loading frequency in the shock tunnel by the hypersonic freestream. The frequency spectrum of the accelerometer signals obtained during the shock tunnel tests had the most prominent frequency components below $1.5 \mathrm{kHz}$, wherein the aerodynamic loading frequency by the hypersonic freestream was in the range of $600 \mathrm{~Hz}-1.5 \mathrm{kHz}$. Hence, the test assembly was apt for the current shock tunnel tests.

2.3. Dynamic Calibration. The system was calibrated for its impulse response function for a range of dynamic input, imparted by an impulse hammer. The impulse hammer used was from PCB Piezotronics (model number 086C02), with a suitable metallic tip to load the model with a requisite impulse. The hammer was equipped with a force sensor that could transmit the force-time history of the applied load. The loading range of the hammer varied from 3 to $444 \mathrm{~N}$. The system was considered to be a linear, time-invariant dynamic system under the given test conditions. The input and output 


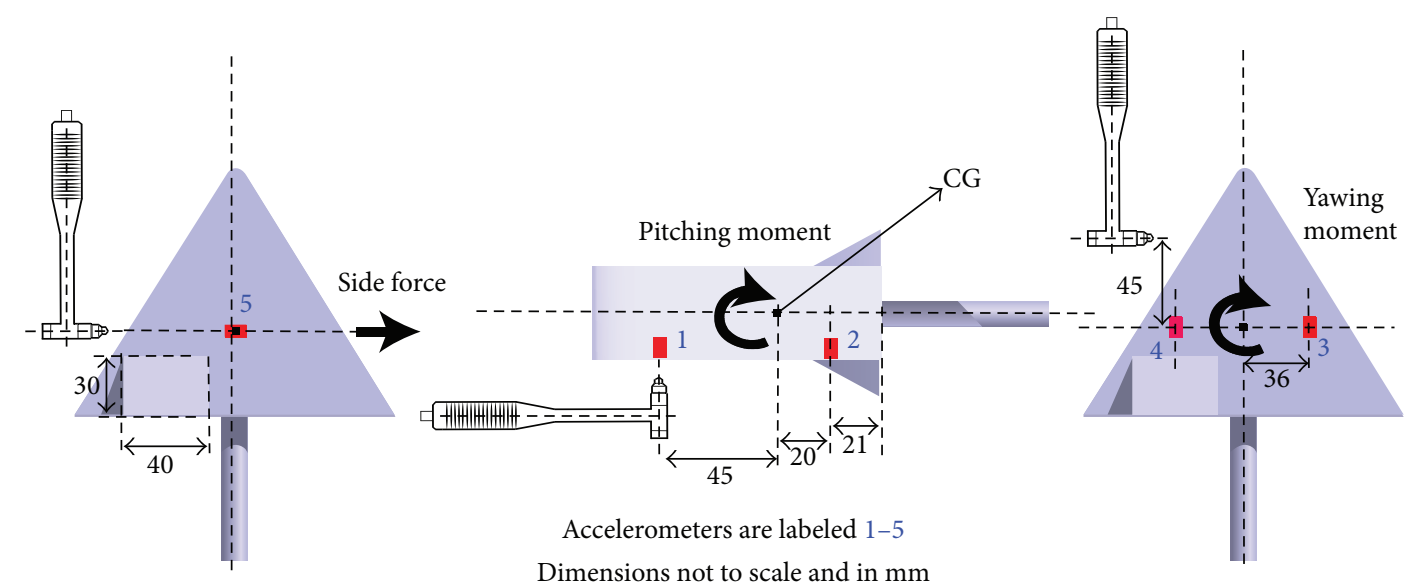

FIGURE 5: Dynamic calibration (impulse loading) procedure for side force, pitching, and yawing moments.
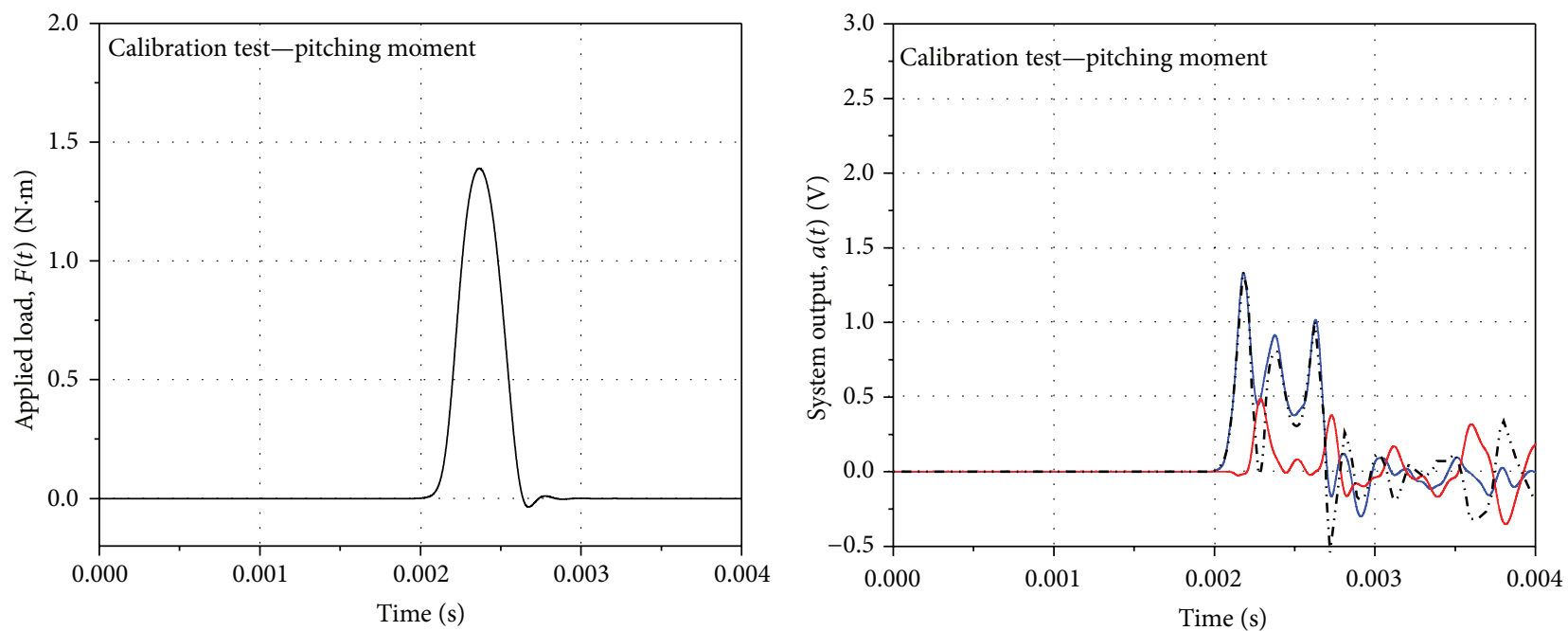

$\begin{array}{ll} & n_{1} \text { (accelerometer 1) } \\ - & n_{2} \text { (accelerometer 2) } \\ \ldots \ldots & \text { pm }=n_{1}-n_{2}\end{array}$

(a)

(b)

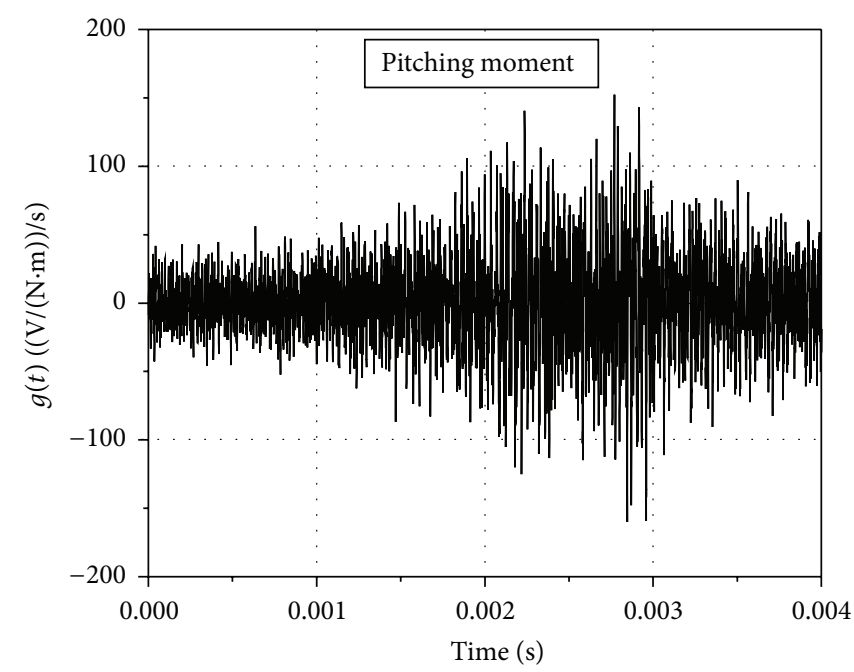

(c)

Figure 6: Typical signals of (a) applied load, (b) system output, and (c) impulse response function obtained during/through the dynamic calibration of the test model. 


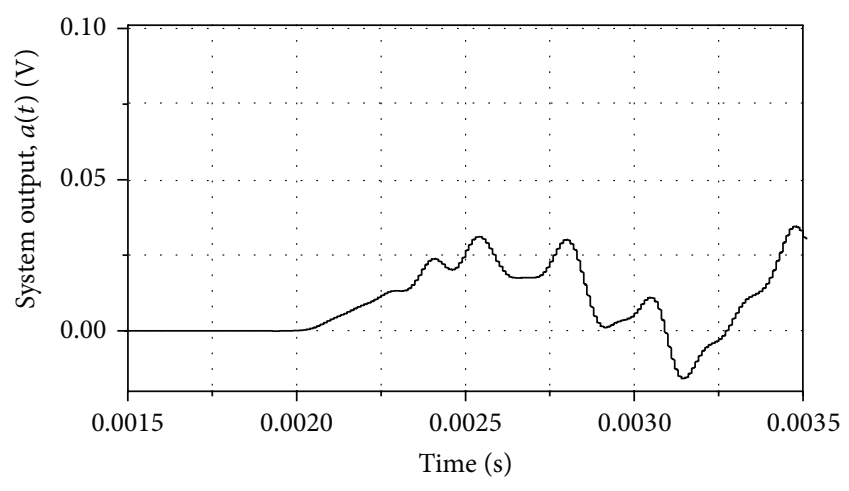

- Accelerometer 5

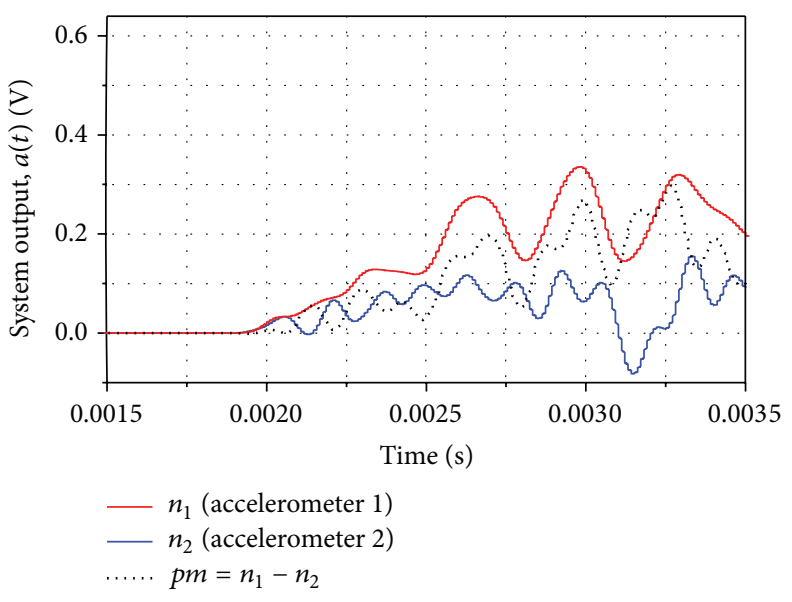

(b)

(a)

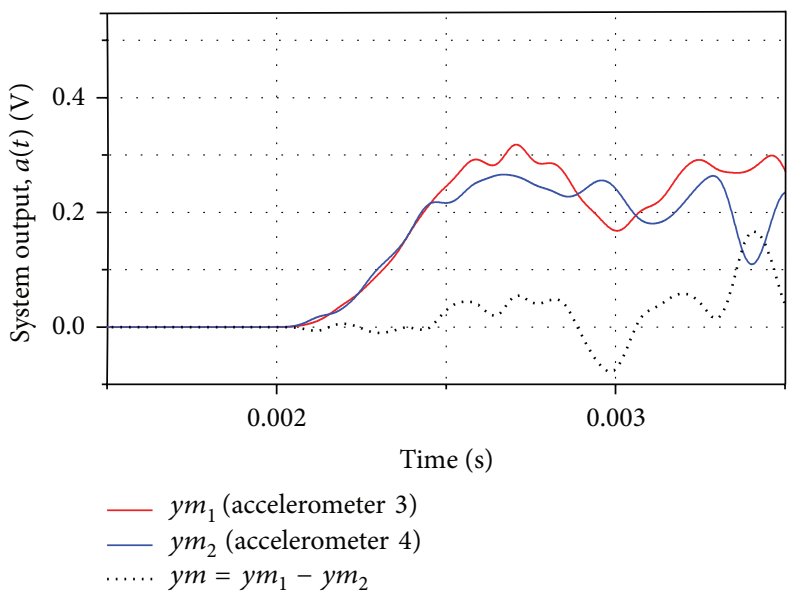

(c)

FIGURE 7: Typical system output obtained during shock tunnel testing. (a) Side force, (b) pitching, and (c) yawing components. AOA $=15^{\circ}$.

of such a system could be correlated using a convolution integral given by

$$
a(t)=\int_{0}^{t} g(t-\tau) F(\tau) d \tau,
$$

where $a(t)$ is the system output (Volt), $g(t)$ is the impulse response function, and $F(t)$ is the applied force (Newton), as depicted by the schematic of linear, time-invariant system in Figure 4.

During the calibration, the system was loaded with impulses of known magnitude that were consistent with the impulse loading by the freestream during the actual tests in the tunnel. The likely magnitude of the aerodynamic load on the model was estimated using the Newtonian theory [10]. The dynamic pressure of the freestream for the operations was $2285 \pm 4 \% \mathrm{~Pa}$, which could load the present test model through $1-15 \mathrm{~N}$, considering all the angles of attack (0-15 degrees), wherein the drag force was the maximum and the side force was the minimum. The model was fixed on its usual mount horizontally during the calibration. The impulse loading procedure for different components of forces is depicted in Figure 5. The location of the CG is indicated in the figure. For the calibration of side force, accelerometer 5 was activated by applying the impulse load along the transverse axis of the model. The pitching was induced by loading the model vertically at the point of location of accelerometer 1 on the bottom plate. Accelerometers 1 and 2 were mounted vertically on either side of the model CG on the bottom plate, along its longitudinal axis to sense pitching. Yawing maneuver was induced by the application of an impulse on a side surface, $45 \mathrm{~mm}$ upstream of the CG. The line of application of the impulse in this case was parallel to the transverse axis of the model. Accelerometers 3 and 4 were mounted on either side of the model CG with their axes parallel to the longitudinal axis of the test model to capture the yawing moment. In the case of moments, the applied load was multiplied by the arm of the moments, which was the perpendicular distance between the model CG and the line of application of the impulse.

The system and hammer output were recorded on a PC based data acquisition system at a frequency of $1 \mathrm{MHz}$. The operating frequency bandwidth of the accelerometers was $1 \mathrm{~Hz}-10 \mathrm{kHz}$, and hence, the output of the system was filtered through a low pass filter of $10 \mathrm{kHz}$ cut-off frequency. 


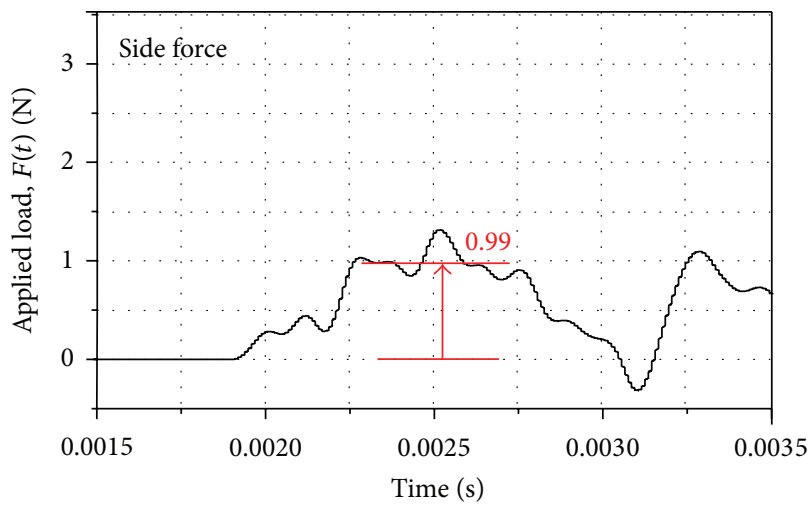

(a)

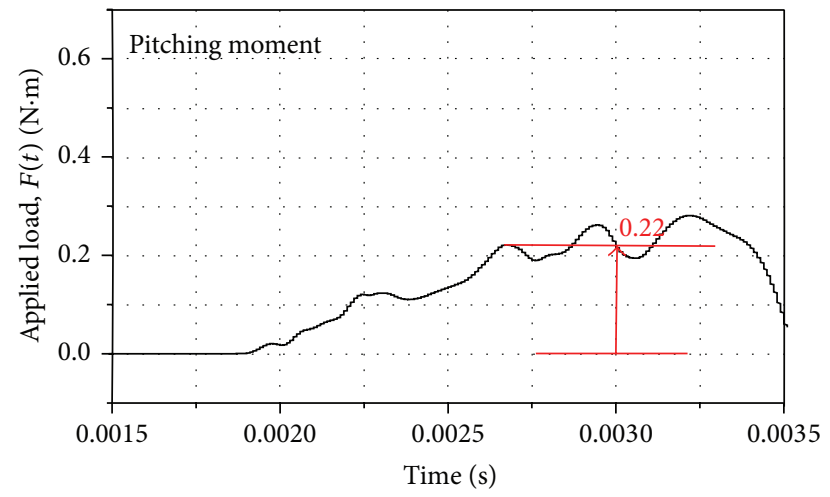

(b)

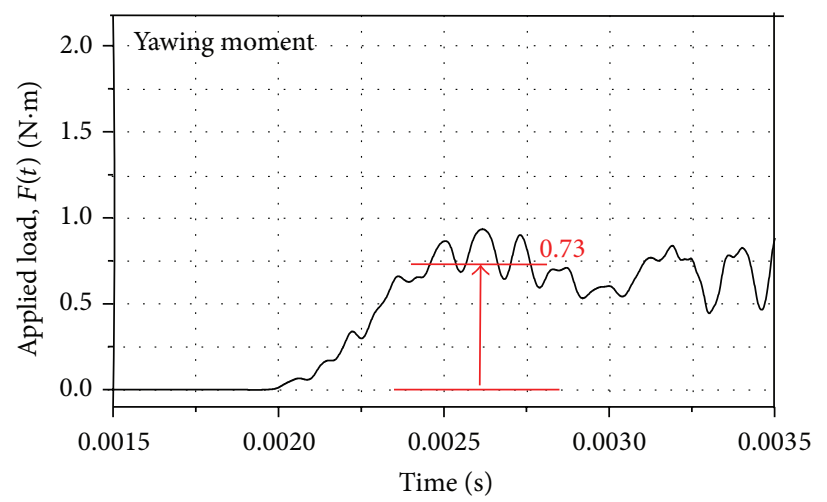

(c)

FIgURE 8: Deconvoluted aerodynamic loads against the system output in Figure 7.

Fast Fourier Transforms (FFT) were carried out on $F(t)$ and $a(t)$ in order to obtain their transformed functions in frequency domain, which were denoted by $F(f)$ and $a(f)$, respectively. The output of the system in the frequency domain is the product of the impulse response function in frequency domain $(g(f))$ and the transformed input, which can be expressed as in (2). The typical signals of applied load, system output, and the resultant system response function obtained during the calibration procedure are presented in Figure 6 for one of the components of the balance (pitching moment). The pulse durations of the input and output are of the order of the actual test duration of the model in the tunnel. The system response function $g(t)$ is presented in time domain and is an average function representing a range of $a(t)$ and $F(t)$ predicted for the present set of experiments (including all the angles of attack) for the pitching moment:

$$
g(f)=\frac{a(f)}{F(f)} .
$$

The outcome of the dynamic calibration is the system's impulse response function $g(f)$, which can be operated on an acquired $a(f)$ to get an unknown $F(f)$ through (2), which indicates that the impulse response function of a linear, time-invariant system is independent of the magnitude of input. An Inverse Fast Fourier Transform (IFFT) on $F(f)$ can yield the unknown input $F(t)$ in time domain, which is the deconvoluted force/moment-time history. The impulse response functions were generated for all the components of forces/moments of interest using the procedure described above and the unknown input of each component during the actual test in the tunnel was obtained. These components were expressed in their nondimensional forms as in (3), where $\mathrm{SF}(t), M(t)$, and $Y(t)$ are the side force, pitching, and yawing moments, respectively; $C_{\mathrm{SF}}, C_{M}$, and $C_{Y}$ are the coefficients of side force, pitching, and yawing moments, respectively, $q_{\infty}$ is the freestream dynamic pressure, and $A$ and $L$ are characteristic reference area and length, respectively:

$$
\begin{aligned}
C_{\mathrm{SF}} & =\frac{\mathrm{SF}(t)}{q_{\infty} \times A}, \\
C_{M} & =\frac{M(t)}{q_{\infty} \times A \times L}, \\
C_{Y} & =\frac{Y(t)}{q_{\infty} \times A \times L} .
\end{aligned}
$$

\section{Results}

The typical signals obtained from the system during the hypersonic flow in the tunnel are presented in Figure 7. These are the model accelerations corresponding to side force, pitching, and yawing moments, respectively. The model angle of attack (AOA) pertinent to the presented signals was $15^{\circ}$. The signals indicate readable amplitudes within the test 


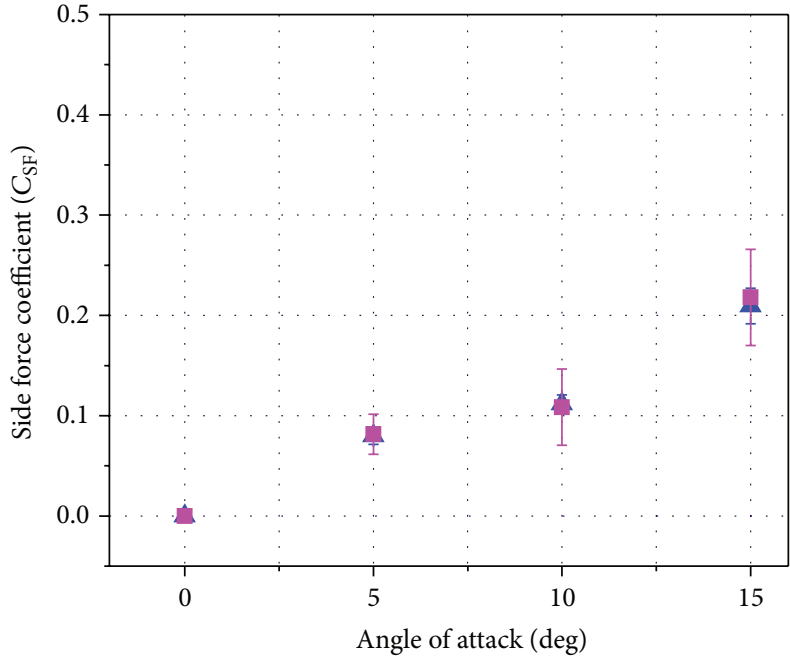

A Balance theory [11]

- Convolution technique

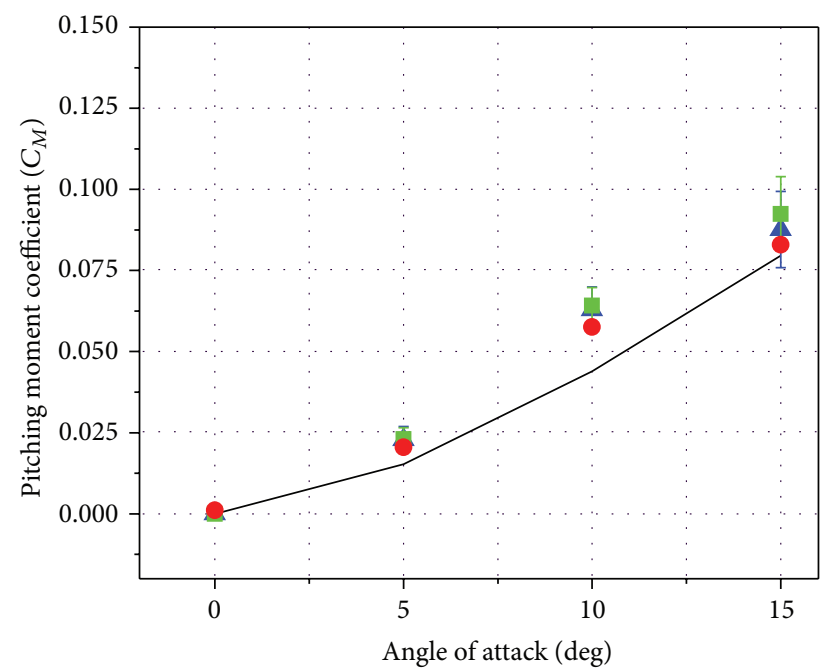

A Balance theory [11]

Pressure measurement [11]

(b)

(a)

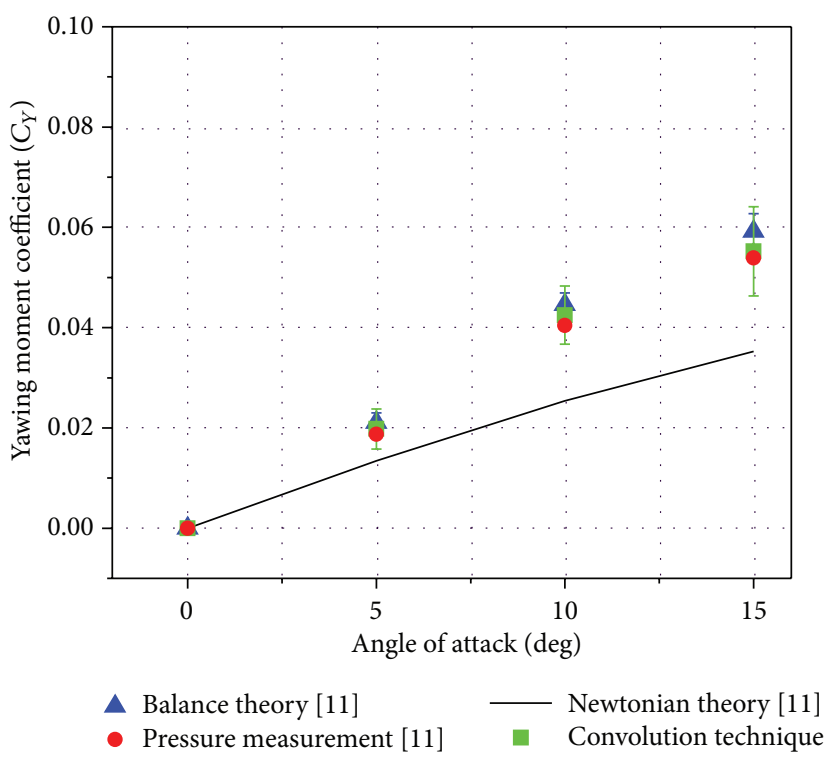

(c)

FIGURE 9: Variation of (a) side force, (b) pitching, and (c) yawing moment coefficients with model AOA. The scatter bars indicate standard deviation.

duration of the tunnel, indicating an apt response of the force balance to the impulse of the hypersonic freestream. The difference of the readings of accelerometers 1 and 2 was the representation of the magnitude of pitching, while the difference of the output of accelerometers 3 and 4 represented the magnitude of yawing of the model. The impulse response functions obtained through the calibration procedure were operated on the above output signals of the system in their frequency domains, as described by (2), and were transformed back into the time domain by IFFT as force-time and moment-time histories, which were the unknown input to the system from the hypersonic freestream in the tunnel. The deconvoluted input/applied load signals of side force, pitching, and yawing moments are presented in Figure 8. The force and moments on the model could be directly read from the signals as indicated by the horizontal markers.

The plots in Figure 9 show the variation of coefficients of side force, pitching, and yawing moments with the angles of incidence of the test model. The data on the aerodynamic moments is of an experimental (convolution) technique adopted in the present study and of an analytical and two experimental methodologies followed previously [11]. The data on the side force is of the present and of a previous (balance theory) experiment, as the side force on the test model could only be measured accurately by the force balance. The agreement among the different sets of experimental data is 
quite close, while the analytical data has a deviation owing to the flow field complexities not built into the analytical method (Newtonian theory). The flow field of the model had severe viscous and strong shock effects on the flaps at higher angles of incidence, which were not taken into account by the Newtonian theory.

The model generated most of the degrees of freedom during its free-flight by virtue of the trailing edge flaps. Rolling, yawing, side force, and, to some extent, pitching were induced on the model by the flaps. In the present study, the side force, pitching, and yawing moments were activated when the model was set at an AOA, as seen in Figure 9. While the pitching and yawing could be brought about merely by the usual AOA (AOA with respect to the longitudinal axis of the model), the side force required the test model to yaw through an angle, to come about. The yaw angle generated was dynamic during the ultrashort test duration and could not accurately be determined as of now, and hence this aspect could not be included in our analytical and local surface (pressure) measurement procedures effectively. Hence, the coefficient of side force presented is of the two experimental procedures carried out in the tunnel at actuals.

The estimated uncertainties in the reported coefficients are $4.31 \%, 4.5 \%$, and $4.33 \%$ for side force, pitching, and yawing moment coefficients, respectively. The uncertainties are attributable to the errors in the sensor sensitivities, derived freestream conditions, system output, and the data acquisition system.

\section{Conclusions}

Side force, pitching, and yawing moments were measured on a blunt, flapped, delta wing at various angles of attack, in an ultrashort duration hypersonic freestream using an accelerometer force balance. A novel, impulse response function based convolution technique was used as the data reduction methodology for deducing the unknown force and moments from the balance output. The impulse response function obtained through the convolution technique eliminates the uncertainties concerning restraint of the model (due to the suspension stiffness), sensor sensitivity, structural gaps, and theoretical assumptions, making it a more reliable data reduction technique. The reduced nondimensional data on the above force/moment components was compared with the results of other techniques, namely, pressure measurement and balance theory, and the agreement has been found encouraging. The validity of the balance is for small, rigid models under impulse loading.

\section{Conflict of Interests}

The authors declare that there is no conflict of interests regarding the publication of this paper.

\section{Acknowledgments}

This work was jointly funded by the Grant-in-Aid no. 12ISROC005 of the ISRO-IITB Space Technology Cell, IIT
Bombay, and the Grant-in-Aid (vide Order no. SR/FST/ETI260/2009 dated 26/02/2010) of the FIST Program of the Department of Science and Technology, New Delhi. The technical contribution by Dr. Sharad Trivedi is immensely acknowledged.

\section{References}

[1] N. Sahoo, K. Suryavamshi, K. P. J. Reddy, and D. J. Mee, "Dynamic force balances for short-duration hypersonic testing facilities," Experiments in Fluids, vol. 38, no. 5, pp. 606-614, 2005.

[2] R. Joarder and G. Jagadeesh, "A new free floating accelerometer balance system for force measurements in shock tunnels," Shock Waves, vol. 13, no. 5, pp. 409-412, 2004.

[3] N. Sahoo, D. R. Mahapatra, G. Jagadeesh, S. Gopalakrishnan, and K. P. J. Reddy, "An accelerometer balance system for measurement of aerodynamic force coefficients over blunt bodies in a hypersonic shock tunnel," Measurement Science and Technology, vol. 14, no. 3, pp. 260-272, 2003.

[4] D. J. Mee, "Dynamic calibration of force balances for impulse hypersonic facilities," Shock Waves, vol. 12, no. 6, pp. 443-455, 2003.

[5] J. Srulijes, P. Gnemmi, K. Runne, and F. Seiler, "High-pressure shock tunnel experiments and CFD calculations on spiketipped blunt bodies," in Proceedings of the 22nd AIAA Aerodynamic Measurement Technology and Ground Testing Conference, AIAA Paper 2002-2918, Seattle, Wash, USA, June 2002.

[6] S. J. Laurence and S. Karl, "An improved visualization-based force-measurement technique for short-duration hypersonic facilities," Experiments in Fluids, vol. 48, no. 6, pp. 949-965, 2010.

[7] M. M. Abdel-Jawad, D. J. Mee, and R. G. Morgan, "New calibration technique for multiple-component stress wave force balances," Review of Scientific Instruments, vol. 78, no. 6, Article ID 065101, 2007.

[8] V. Menezes and S. Bhat, "A coaxial thermocouple for shock tunnel applications," Review of Scientific Instruments, vol. 81, no. 10, Article ID 104905, 2010.

[9] P. Singh, S. Trivedi, V. Menezes, and H. Hosseini, "Dynamic calibration and validation of an accelerometer force balance for hypersonic lifting models," The Scientific World Journal, vol. 2014, Article ID 813759, 8 pages, 2014.

[10] R. W. Truitt, Hypersonic Aerodynamics, Ronald Press, New York, NY, USA, 1959.

[11] S. Trivedi and V. Menezes, "Measurement of yaw, pitch and side-force on a lifting model in a hypersonic shock tunnel," Measurement, vol. 45, no. 7, pp. 1755-1764, 2012. 

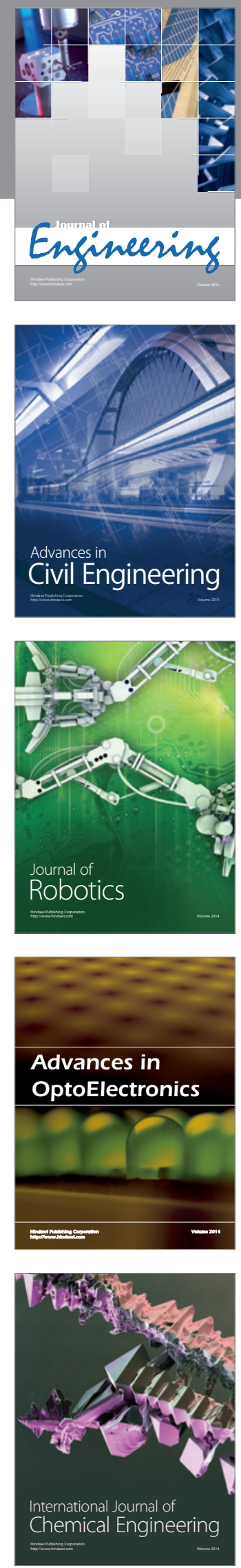

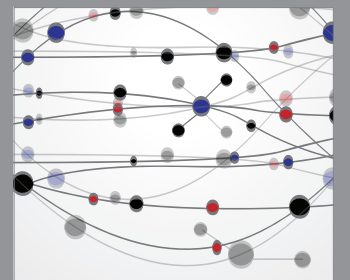

The Scientific World Journal
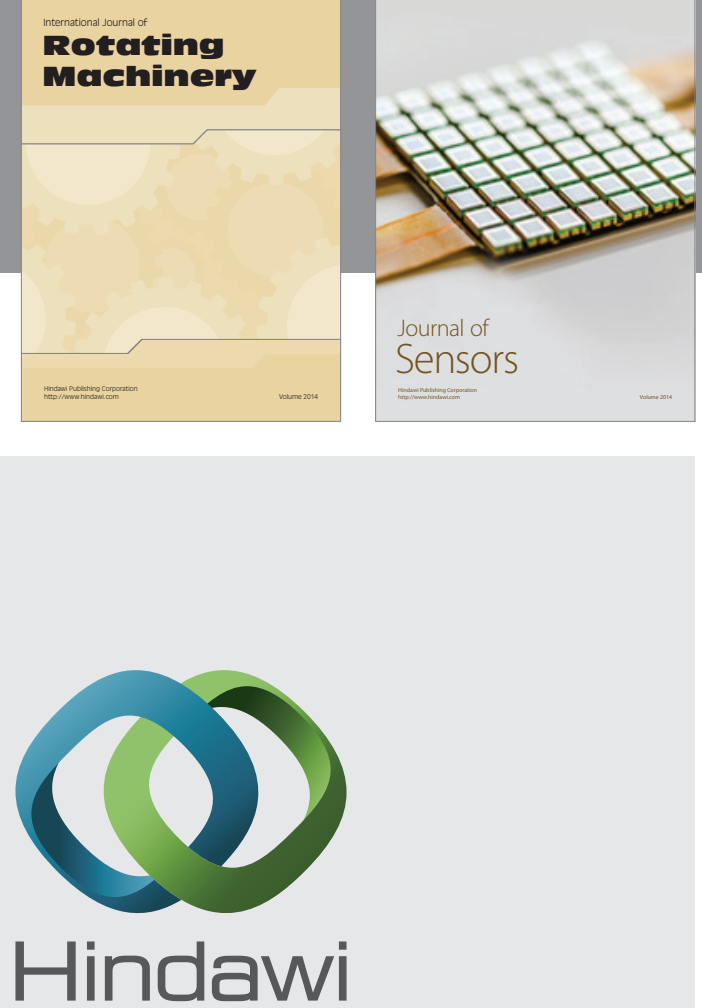

Submit your manuscripts at http://www.hindawi.com
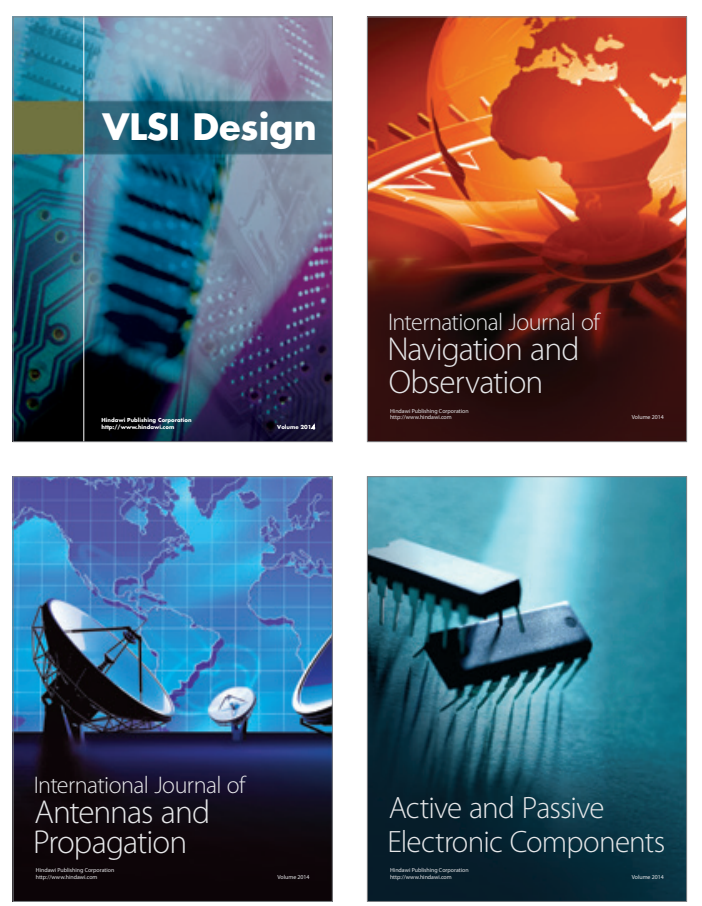
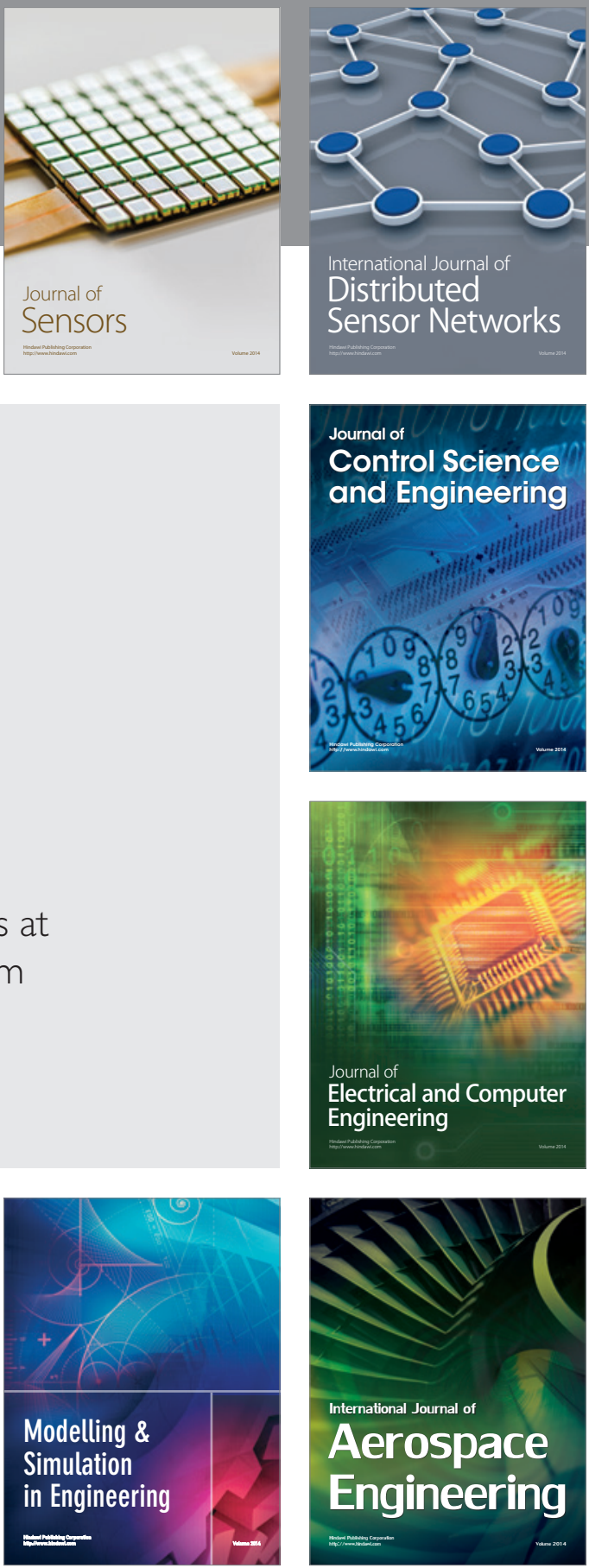

Journal of

Control Science

and Engineering
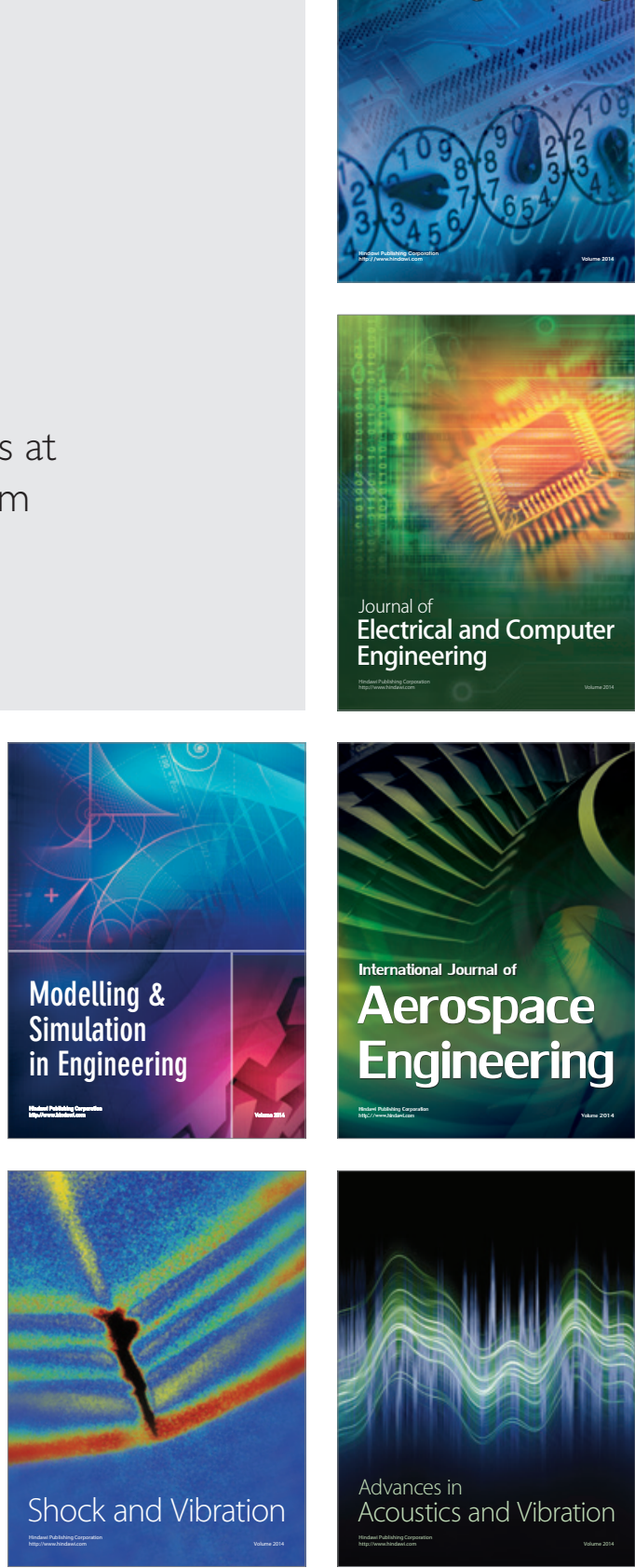